Probl. Anal. Issues Anal. Vol. 5 (23), No. 1, 2016, pp. 3-20

DOI: $10.15393 /$ j3.art.2016.3071

UDC 517.51, 517.38

Z. LIU

\title{
ON INEQUALITIES OF HERMITE-HADAMARD TYPE INVOLVING AN S-CONVEX FUNCTION WITH APPLICATIONS
}

\begin{abstract}
Motivated by a recent paper, the author provides some new integral inequalities of Hermite-Hadamard type involving the product of an s-convex function and a symmetric function and applies these new established inequalities to construct inequalities for special means.
\end{abstract}

Key words: Hermite-Hadamard's integral inequality, s-convex function, symmetric function, Hölder inequality, mean

2010 Mathematical Subject Classification: 26D15

1. Introduction. Let $f: I \subseteq \mathbf{R} \rightarrow \mathbf{R}$ be a convex function on an interval $I$ of real numbers and $a, b \in I$ with $a<b$. The inequality

$$
f\left(\frac{a+b}{2}\right) \leq \frac{1}{b-a} \int_{a}^{b} f(x) d x \leq \frac{f(a)+f(b)}{2}
$$

is well known in literature as the Hermite-Hadamard inequality for convex functions.

We recall that Hudzik and Maligranda in [1] defined a function $f:[0, \infty) \rightarrow \mathbf{R}$ to be called s-convex in the second sense if

$$
f(\lambda x+(1-\lambda) y) \leq \lambda^{s} f(x)+(1-\lambda)^{s} f(y)
$$

holds for all $x, y \in[0, \infty), \lambda \in[0,1]$ and for some fixed $s \in(0,1]$. The class of s-convex functions in the second sense is usually denoted with $K_{s}^{2}$. It can be easily seen that for $s=1 \mathrm{~s}$-convexity reduces to ordinary convexity of functions defined on $[0, \infty)$. It is proved in [1] that all functions from $K_{s}^{2}, s \in(0,1)$ are nonnegative. 
Example 1. [1] Let $s \in(0,1)$ and $a, b, c \in \mathbf{R}$. Define the function $f:[0, \infty) \rightarrow \mathbf{R}$ as

$$
f(t)= \begin{cases}a, & t=0 \\ b t^{s}+c, & t>0\end{cases}
$$

It can be easily checked that

(i) if $b \geq 0$ and $0 \leq c \leq a$, then $f \in K_{s}^{2}$,

(ii) if $b>0$ and $c<0$, then $f \notin K_{s}^{2}$.

In the recent paper [2], Hua et al established the following integral inequalities of Hermite-Hadamard type involving the product of an sconvex function and a symmetric function.

Theorem 1.1-1.3. [2, Theorem 3.1, 3.2, 3.5.] Let $f: I \subseteq \mathbf{R} \rightarrow \mathbf{R}$ be differentiable on int $I$ and $g:[a, b] \rightarrow[0, \infty)$ be continuous and symmetric to $\frac{a+b}{2}$ for $a, b \in I$ with $a<b$ such that $f^{\prime} \in L^{1}[a, b]$. If $\left|f^{\prime}\right|^{q}$ is s-convex on $[a, b]$ for $q \geq 1$ and some fixed $s \in(0,1]$, then

$$
\left|\frac{f(a)+f(b)}{2} \int_{a}^{b} g(x) d x-\int_{a}^{b} f(x) g(x) d x\right| \leq \gamma,
$$

where $\gamma$ is the minimum of the following three values:

$$
\begin{gathered}
\frac{(b-a)^{2}}{8}\left[\frac{2^{1-s}}{(s+1)(s+2)}\right]^{1 / q}\|g\|_{\infty}\left\{\left[\left(1+s 2^{s+1}\right)\left|f^{\prime}(a)\right|^{q}+\left|f^{\prime}(b)\right|^{q}\right]^{1 / q}+\right. \\
\left.+\left[\left|f^{\prime}(a)\right|^{q}+\left(1+s 2^{s+1}\right)\left|f^{\prime}(b)\right|^{q}\right]^{1 / q}\right\}, \\
\frac{(b-a)^{2}}{8}\left[\frac{2}{(s+1)(s+2)}\right]^{1 / q}|| g \|_{\infty}\left\{\left[(s+1)\left|f^{\prime}(a)\right|^{q}+\left|f^{\prime}\left(\frac{a+b}{2}\right)\right|^{q}\right]^{1 / q}+\right. \\
\left.+\left[\left|f^{\prime}\left(\frac{a+b}{2}\right)\right|^{q}+(s+1)\left|f^{\prime}(b)\right|^{q}\right]^{1 / q}\right\},
\end{gathered}
$$

and

$$
\frac{b-a}{2}\left[\frac{\left|f^{\prime}(a)\right|^{q}+\left|f^{\prime}(b)\right|^{q}}{2}\right]^{1 / q} \int_{0}^{1}\left[\int_{L(t)}^{U(t)} g(x) d x\right] d t .
$$

Theorem 1.4-1.6. [2, Theorem 3.3, 3.4, 3.6.] Let $f: I \subseteq \mathbf{R} \rightarrow \mathbf{R}$ be differentiable on int $I$ and $g:[a, b] \rightarrow[0, \infty)$ be continuous and symmetric to $\frac{a+b}{2}$ for $a, b \in I$ with $a<b$. If $\left|f^{\prime}\right|^{q}$ for $q>1$ is convex on $[a, b]$, then

$$
\left|\frac{f(a)+f(b)}{2} \int_{a}^{b} g(x) d x-\int_{a}^{b} f(x) g(x) d x\right| \leq \delta,
$$


where $\delta$ is the minimum of the following three values:

$$
\begin{gathered}
\frac{(b-a)^{2}}{4}\left(\frac{q-1}{2 q-1}\right)^{1-1 / q}\left[\frac{1}{2^{s}(s+1)}\right]^{1 / q}\|g\|_{\infty}\left\{\left[\left(2^{s+1}-1\right)\left|f^{\prime}(a)\right|^{q}+\right.\right. \\
\left.\left.+\left|f^{\prime}(b)\right|^{q}\right]^{1 / q}+\left[\left|f^{\prime}(a)\right|^{q}+\left(2^{s+1}-1\right)\left|f^{\prime}(b)\right|^{q}\right]^{1 / q}\right\}, \\
\frac{(b-a)^{2}}{4(s+1)^{1 / q}}\left(\frac{q-1}{2 q-1}\right)^{1-1 / q}\|g\|_{\infty}\left\{\left[\left|f^{\prime}(a)\right|^{q}+\left|f^{\prime}\left(\frac{a+b}{2}\right)\right|^{q}\right]^{1 / q}+\right. \\
\left.+\left[\left|f^{\prime}\left(\frac{a+b}{2}\right)\right|^{q}+\left|f^{\prime}(b)\right|^{q}\right]^{1 / q}\right\},
\end{gathered}
$$

and

$$
\begin{gathered}
\frac{b-a}{4}\left\{\left(\frac{3\left|f^{\prime}(a)\right|^{q}+\left|f^{\prime}(b)\right|^{q}}{4}\right)^{1 / q}+\left(\frac{\left|f^{\prime}(a)\right|^{q}+3\left|f^{\prime}(b)\right|^{q}}{4}\right)^{1 / q}\right\} \times \\
\times\left[\int_{0}^{1}\left[\int_{L(t)}^{U(t)} g(x) d x\right]^{q /(q-1)} d t\right]^{1-1 / q} .
\end{gathered}
$$

Within them,

$$
L(t)=\frac{1+t}{2} a+\frac{1-t}{2} b=t a+(1-t) \frac{a+b}{2}
$$

and

$$
U(t)=\frac{1-t}{2} a+\frac{1+t}{2} b=t b+(1-t) \frac{a+b}{2} .
$$

It should be noticed that we here have improved the expression of Theorem 3.6 in [2].

In this work, corresponding to Theorems 1.1-1.6, we will further establish some integral inequalities of Hermite-Hadamard type involving the product of an s-convex function and a symmetric function in two different ways. Finally, applications to some special means of positive real numbers are considered.

\section{Main Results.}

Lemma 2.1. (see [3]) Let $f: I \subseteq \mathbf{R} \rightarrow \mathbf{R}$ be differentiable on int $I$ and $g:[a, b] \rightarrow[0, \infty)$ be continuous and symmetric to $\frac{a+b}{2}$ for $a, b \in I$ with 
$a<b$. If $f^{\prime} \in L^{1}[a, b]$, then

$$
\begin{aligned}
& \int_{a}^{b} f(x) g(x) d x-f\left(\frac{a+b}{2}\right) \int_{a}^{b} g(x) d x= \\
= & \frac{b-a}{2} \int_{0}^{1}\left[\int_{a}^{L(t)} g(x) d x\right]\left[f^{\prime}(U(t))-f^{\prime}(L(t))\right] d t,
\end{aligned}
$$

where $L$ and $U$ are defined by (1) and (2). In particular, we then have

$$
\begin{gathered}
\left|\int_{a}^{b} f(x) g(x) d x-f\left(\frac{a+b}{2}\right) \int_{a}^{b} g(x) d x\right| \leq \\
\leq \frac{b-a}{2} \int_{0}^{1}\left[\int_{a}^{L(t)} g(x) d x\right]\left[\left|f^{\prime}(L(t))\right|+\left|f^{\prime}(U(t))\right|\right] d t
\end{gathered}
$$

and

$$
\begin{aligned}
& \left|\int_{a}^{b} f(x) g(x) d x-f\left(\frac{a+b}{2}\right) \int_{a}^{b} g(x) d x\right| \leq \\
\leq & \frac{(b-a)^{2}}{4}\|g\|_{\infty} \int_{0}^{1}(1-t)\left[\mid f^{\prime}\left(L(t)|+| f^{\prime}(U(t) \mid] d t,\right.\right.
\end{aligned}
$$

where $\|g\|_{\infty}=\sup _{t \in[a, b]} g(t)$.

Proof. Since $g(x)$ is symmetric to $\frac{a+b}{2}$, then $\int_{a}^{L(t)} g(x) d x=\int_{U(t)}^{b} g(x) d x$ for all $t \in[0,1]$. So we have

$$
\begin{aligned}
& \int_{0}^{1}\left[\int_{a}^{L(t)} g(x) d x\right] f^{\prime}(L(t)) d t=\frac{2}{a-b} \int_{0}^{1}\left[\int_{a}^{L(t)} g(x) d x\right] d[f(L(t))]= \\
& =\frac{2}{a-b}\left\{\left.\left[\int_{a}^{L(t)} g(x) d x\right] f(L(t))\right|_{0} ^{1}+\frac{b-a}{2} \int_{0}^{1} f(L(t)) g(L(t)) d t\right\}=
\end{aligned}
$$




$$
=\frac{2}{a-b}\left\{-f\left(\frac{a+b}{2}\right) \int_{a}^{\frac{a+b}{2}} g(x) d x+\int_{a}^{\frac{a+b}{2}} f(x) g(x) d x\right\}
$$

and

$$
\begin{gathered}
\int_{0}^{1}\left[\int_{a}^{L(t)} g(x) d x\right] f^{\prime}(U(t)) d t=\int_{0}^{1}\left[\int_{U(t)}^{b} g(x) d x\right] f^{\prime}(U(t)) d t= \\
=\frac{2}{b-a} \int_{0}^{1}\left[\int_{U(t)}^{b} g(x) d x\right] d[f(U(t))]= \\
=\frac{2}{b-a}\left\{\left.\left[\int_{U(t)}^{b} g(x) d x\right] f(U(t))\right|_{0} ^{1}+\frac{b-a}{2} \int_{0}^{1} f(U(t)) g(U(t)) d t\right\}= \\
=\frac{2}{b-a}\left\{-f\left(\frac{a+b}{2}\right) \int_{\frac{a+b}{2}}^{b} g(x) d x+\int_{\frac{a+b}{2}}^{b} f(x) g(x) d x\right\} .
\end{gathered}
$$

Consequently, inequality (3) follows from (6) and (7), and Lemma 2.1 is thus proved.

Theorem 2.1. Let $f: I \subseteq \mathbf{R} \rightarrow \mathbf{R}$ be differentiable on int $I$ and $g:[a, b] \rightarrow[0, \infty)$ be continuous and symmetric to $\frac{a+b}{2}$ for $a, b \in I$ with $a<b$ such that $f^{\prime} \in L^{1}[a, b]$. If $\left|f^{\prime}\right|^{q}$ is s-convex on $[a, b]$ for $q \geq 1$ and some fixed $s \in(0,1]$, then

$$
\begin{gathered}
\left|\int_{a}^{b} f(x) g(x) d x-f\left(\frac{a+b}{2}\right) \int_{a}^{b} g(x) d x\right| \leq \\
\leq \frac{(b-a)^{2}}{8}\left(\frac{2^{1-s}}{s+2}\right)^{1 / q}\|g\|_{\infty}\left\{\left[\frac{2^{s+2}-s-3}{s+1}\left|f^{\prime}(a)\right|^{q}+\left|f^{\prime}(b)\right|^{q}\right]^{1 / q}+\right. \\
\left.+\left[\left|f^{\prime}(a)\right|^{q}+\frac{2^{s+2}-s-3}{s+1}\left|f^{\prime}(b)\right|^{q}\right]^{1 / q}\right\} .
\end{gathered}
$$


Proof. Notice that $\left|f^{\prime}\right|^{q}$ is s-convex on $[a, b]$, by (5) in Lemma 2.1 with the first equality in (1) and (2), and using the Hölder inequality, we have

$$
\begin{aligned}
& \left|\int_{a}^{b} f(x) g(x) d x-f\left(\frac{a+b}{2}\right) \int_{a}^{b} g(x) d x\right| \leq \\
& \leq \frac{(b-a)^{2}}{4}\|g\|_{\infty} \int_{0}^{1}(1-t)\left[\left|f^{\prime}(L(t))\right|+\left|f^{\prime}(U(t))\right|\right] d t \leq \\
& \leq \frac{(b-a)^{2}}{4}\|g\|_{\infty}\left[\int_{0}^{1}(1-t) d t\right]^{1-1 / q} \times \\
& \times\left\{\left[\int_{0}^{1}(1-t)\left(\left(\frac{1+t}{2}\right)\right)^{s}\left|f^{\prime}(a)\right|^{q}+\left(\frac{1-t}{2}\right)^{s}\left|f^{\prime}(b)\right|^{q}\right) d t\right]^{1 / q}+ \\
& \left.+\left[\int_{0}^{1}(1-t)\left(\left(\frac{1-t}{2}\right)^{s}\left|f^{\prime}(a)\right|^{q}+\left(\frac{1+t}{2}\right){ }^{s}\left|f^{\prime}(b)\right|^{q}\right) d t\right]^{1 / q}\right\}=\frac{(b-a)^{2}}{4} \times \\
& \times\|g\|_{\infty}\left(\frac{1}{2}\right)^{1-1 / q}\left\{\left[\frac{2^{s+2}-s-3}{2^{s}(s+1)(s+2)}\left|f^{\prime}(a)\right|^{q}+\frac{1}{2^{s}(s+2)}\left|f^{\prime}(b)\right|^{q}\right]^{1 / q}+\right. \\
& \left.+\left[\frac{1}{2^{s}(s+2)}\left|f^{\prime}(a)\right|^{q}+\frac{2^{s+2}-s-3}{2^{s}(s+1)(s+2)}\left|f^{\prime}(b)\right|^{q}\right]^{1 / q}\right\}= \\
& =\frac{(b-a)^{2}}{8}\|g\|_{\infty}\left(\frac{2^{1-s}}{s+2}\right)^{1 / q}\left\{\left[\frac{2^{s+2}-s-3}{s+1}\left|f^{\prime}(a)\right|^{q}+\right.\right. \\
& \left.\left.+\left|f^{\prime}(b)\right|^{q}\right]^{1 / q}+\left[\left|f^{\prime}(a)\right|^{q}+\frac{2^{s+2}-s-3}{s+1}\left|f^{\prime}(b)\right|^{q}\right]^{1 / q}\right\} .
\end{aligned}
$$

Inequality (8) follows, and Theorem 2.1 is proved.

Corollary 2.1.1. Under conditions of Theorem 2.1,

(i) if $q=1$, then

$$
\left|\int_{a}^{b} f(x) g(x) d x-f\left(\frac{a+b}{2}\right) \int_{a}^{b} g(x) d x\right| \leq
$$




$$
\leq \frac{(b-a)^{2}}{2}\|g\|_{\infty}\left[\frac{2^{s+1}-1}{2^{s}(s+1)(s+2)}\right]\left[\left|f^{\prime}(a)\right|+\left|f^{\prime}(b)\right|\right]
$$

(ii) if $q=1$ and $s=1$, we have

$\left|\int_{a}^{b} f(x) g(x) d x-f\left(\frac{a+b}{2}\right) \int_{a}^{b} g(x) d x\right| \leq \frac{(b-a)^{2}}{8}\|g\|_{\infty}\left[\left|f^{\prime}(a)\right|+\left|f^{\prime}(b)\right|\right]$.

Corollary 2.1.2. Under conditions of Theorem 2.1,

(i) if $q=1$ and $g(x)=1$ for $x \in[a, b]$, then

$$
\left|\frac{1}{b-a} \int_{a}^{b} f(x) d x-f\left(\frac{a+b}{2}\right)\right| \leq \frac{\left(2^{s+1}-1\right)(b-a)}{2^{s+1}(s+1)(s+2)}\left[\left|f^{\prime}(a)\right|+\left|f^{\prime}(b)\right|\right] ;
$$

(ii) if $q=1, g(x)=1$ for $x \in[a, b]$, and $s=1$, we have

$$
\left|\frac{1}{b-a} \int_{a}^{b} f(x) d x-f\left(\frac{a+b}{2}\right)\right| \leq \frac{b-a}{8}\left[\left|f^{\prime}(a)\right|+\left|f^{\prime}(b)\right|\right]
$$

and it should be noticed that inequality (9) first appeared in [4].

Theorem 2.2. Let $f: I \subseteq \mathbf{R} \rightarrow \mathbf{R}$ be differentiable on int $I$ and $g:[a, b] \rightarrow[0, \infty)$ be continuous and symmetric to $\frac{a+b}{2}$ for $a, b \in I$ with $a<b$ such that $f^{\prime} \in L^{1}[a, b]$. If $\left|f^{\prime}\right|^{q}$ is s-convex on $[a, b]$ for $q \geq 1$ and some fixed $s \in(0,1]$, then

$$
\begin{gathered}
\left|\int_{a}^{b} f(x) g(x) d x-f\left(\frac{a+b}{2}\right) \int_{a}^{b} g(x) d x\right| \leq \frac{(b-a)^{2}}{8}\left[\frac{2}{(s+1)(s+2)}\right]^{1 / q} \times \\
\times\|g\|_{\infty}\left\{\left[\left|f^{\prime}(a)\right|^{q}+(s+1)\left|f^{\prime}\left(\frac{a+b}{2}\right)\right|^{q}\right]^{1 / q}+\right. \\
\left.+\left[(s+1)\left|f^{\prime}\left(\frac{a+b}{2}\right)\right|^{q}+\left|f^{\prime}(b)\right|^{q}\right]^{1 / q}\right\} .
\end{gathered}
$$


Proof. Notice that $\left|f^{\prime}\right|^{q}$ is s-convex on $[a, b]$, by (5) in Lemma 2.1 with the second equality in (1) and (2), and using the Hölder inequality in a different way, we have

$$
\begin{aligned}
& \left|\int_{a}^{b} f(x) g(x) d x-f\left(\frac{a+b}{2}\right) \int_{a}^{b} g(x) d x\right| \leq \\
& \leq \frac{(b-a)^{2}}{4}\|g\|_{\infty} \int_{0}^{1}(1-t)\left[\left|f^{\prime}(L(t))\right|+\left|f^{\prime}(U(t))\right|\right] d t= \\
& =\frac{(b-a)^{2}}{4}\|g\|_{\infty} \int_{0}^{1}(1-t)\left[\left|f^{\prime}\left(t a+(1-t) \frac{a+b}{2}\right)\right|+\right. \\
& \left.+\left|f^{\prime}\left(t b+(1-t) \frac{a+b}{2}\right)\right|\right] d t \leq \frac{(b-a)^{2}}{4}\|g\|_{\infty}\left[\int_{0}^{1}(1-t) d t\right]^{1-1 / q} \times \\
& \times\left\{\left[\int_{0}^{1}(1-t)\left(t^{s}\left|f^{\prime}(a)\right|^{q}+(1-t)^{s}\left|f^{\prime}\left(\frac{a+b}{2}\right)\right|^{q}\right) d t\right]^{1 / q}+\right. \\
& \left.+\left[\int_{0}^{1}(1-t)\left((1-t)^{s}\left|f^{\prime}\left(\frac{a+b}{2}\right)\right|^{q}+t^{s}\left|f^{\prime}(b)\right|^{q}\right) d t\right]^{1 / q}\right\}=\frac{(b-a)^{2}}{4} \times \\
& \times\|g\|_{\infty}\left(\frac{1}{2}\right)^{1-1 / q}\left\{\left[\frac{1}{(s+1)(s+2)}\left|f^{\prime}(a)\right|^{q}+\frac{1}{s+2}\left|f^{\prime}\left(\frac{a+b}{2}\right)\right|^{q}\right]^{1 / q}+\right. \\
& \left.+\left[\frac{1}{s+2}\left|f^{\prime}\left(\frac{a+b}{2}\right)\right|^{q}+\frac{1}{(s+1)(s+2)}\left|f^{\prime}(b)\right|^{q}\right]^{1 / q}\right\}=\frac{(b-a)^{2}}{8} \times \\
& \times\|g\|_{\infty}\left[\frac{2}{(s+1)(s+2)}\right]^{1 / q}\left\{\left[\left|f^{\prime}(a)\right|^{q}+(s+1)\left|f^{\prime}\left(\frac{a+b}{2}\right)\right|^{q}\right]^{1 / q}+\right. \\
& \left.+\left[(s+1)\left|f^{\prime}\left(\frac{a+b}{2}\right)\right|^{q}+\left|f^{\prime}(b)\right|^{q}\right]^{1 / q}\right\} .
\end{aligned}
$$

Inequality (10) follows, and Theorem 2.2 is proved. 
Corollary 2.2.1. Under conditions of Theorem 2.2,

(i) if $q=1$, then

$$
\begin{gathered}
\left|\int_{a}^{b} f(x) g(x) d x-f\left(\frac{a+b}{2}\right) \int_{a}^{b} g(x) d x\right| \leq \\
\leq \frac{(b-a)^{2}}{4(s+1)(s+2)}|| g \|_{\infty}\left[\left|f^{\prime}(a)\right|+2(s+1)\left|f^{\prime}\left(\frac{a+b}{2}\right)\right|+\left|f^{\prime}(b)\right|\right] ;
\end{gathered}
$$

(ii) if $q=1$ and $s=1$, we have

$$
\begin{gathered}
\left|\int_{a}^{b} f(x) g(x) d x-f\left(\frac{a+b}{2}\right) \int_{a}^{b} g(x) d x\right| \leq \\
\leq \frac{(b-a)^{2}}{24}\|g\|_{\infty}\left[\left|f^{\prime}(a)\right|+4\left|f^{\prime}\left(\frac{a+b}{2}\right)\right|+\left|f^{\prime}(b)\right|\right] .
\end{gathered}
$$

Corollary 2.2.2. Under conditions of Theorem 2.2,

(i) if $q=1$ and $g(x)=1$ for $x \in[a, b]$, then

$$
\begin{gathered}
\left|\frac{1}{b-a} \int_{a}^{b} f(x) d x-f\left(\frac{a+b}{2}\right)\right| \leq \\
\leq \frac{b-a}{4(s+1)(s+2)}\left[\left|f^{\prime}(a)\right|+2(s+1)\left|f^{\prime}\left(\frac{a+b}{2}\right)\right|+\left|f^{\prime}(b)\right|\right] ;
\end{gathered}
$$

(ii) if $q=1, g(x)=1$ for $x \in[a, b]$, and $s=1$, we have

$$
\begin{gathered}
\left|\frac{1}{b-a} \int_{a}^{b} f(x) d x-f\left(\frac{a+b}{2}\right)\right| \leq \\
\leq \frac{b-a}{24}\left[\left|f^{\prime}(a)\right|+4\left|f^{\prime}\left(\frac{a+b}{2}\right)\right|+\left|f^{\prime}(b)\right|\right],
\end{gathered}
$$

and it should be noticed that inequality (11) can also be derived from (2.8) of [5].

Theorem 2.3. Let $f: I \subseteq \mathbf{R} \rightarrow \mathbf{R}$ be differentiable on int $I$ and $g:[a, b] \rightarrow[0, \infty)$ be continuous and symmetric to $\frac{a+b}{2}$ for $a, b \in I$ with 
$a<b$ such that $f^{\prime} \in L^{1}[a, b]$. If $\left|f^{\prime}\right|^{q}$ is s-convex on $[a, b]$ for $q>1$ and some fixed $s \in(0,1]$, then

$$
\begin{gathered}
\left|\int_{a}^{b} f(x) g(x) d x-f\left(\frac{a+b}{2}\right) \int_{a}^{b} g(x) d x\right| \leq \\
\leq \frac{(b-a)^{2}}{4}\|g\|_{\infty}\left(\frac{q-1}{2 q-1}\right)^{1-1 / q}\left[\frac{1}{2^{s}(s+1)}\right]^{1 / q}\left\{\left[\left(2^{s+1}-1\right)\left|f^{\prime}(a)\right|^{q}+\right.\right. \\
\left.\left.+\left|f^{\prime}(b)\right|^{q}\right]^{1 / q}+\left[\left|f^{\prime}(a)\right|^{q}+\left(2^{s+1}-1\right)\left|f^{\prime}(b)\right|^{q}\right]^{1 / q}\right\}
\end{gathered}
$$

Proof. Notice that $\left|f^{\prime}\right|^{q}$ is s-convex on $[a, b]$, by (5) in Lemma 2.1 with the first equality in (1) and (2), and using the Hölder inequality, we have

$$
\begin{gathered}
\left|\int_{a}^{b} f(x) g(x) d x-f\left(\frac{a+b}{2}\right) \int_{a}^{b} g(x) d x\right| \leq \\
\leq \frac{(b-a)^{2}}{4}\|g\|_{\infty} \int_{0}^{1}(1-t)\left[\left|f^{\prime}(L(t))\right|+\left|f^{\prime}(U(t))\right|\right] d t \leq \\
\leq \frac{(b-a)^{2}}{4}|| g \|_{\infty}\left[\int_{0}^{1}(1-t)^{q / q-1} d t\right]^{1-1 / q} \times \\
\times\left\{\int_{0}^{1}\left(\left(\frac{1+t}{2}\right)^{s}\left|f^{\prime}(a)\right|^{q}+\left(\frac{1-t}{2}\right)^{s}\left|f^{\prime}(b)\right|^{q}\right) d t\right]^{1 / q}+ \\
\left.+\left[\int_{0}^{1}\left(\left(\frac{1-t}{2}\right)^{s}\left|f^{\prime}(a)\right|^{q}+\left(\frac{1+t}{2}\right)^{s}\left|f^{\prime}(b)\right|^{q}\right) d t\right]^{1 / q}\right\}= \\
=\frac{(b-a)^{2}}{4}\|g\|_{\infty}\left(\frac{q-1}{2 q-1}\right)^{1-1 / q}\left[\frac{1}{2^{s}(s+1)}\right]^{1 / q}\left\{\left[\left(2^{s+1}-1\right)\left|f^{\prime}(a)\right|^{q}+\right.\right. \\
\left.\left.+\left|f^{\prime}(b)\right|^{q}\right]^{1 / q}+\left[\left|f^{\prime}(a)\right|^{q}+\left(2^{s+1}-1\right)\left|f^{\prime}(b)\right|^{q}\right]^{1 / q}\right\} .
\end{gathered}
$$


Inequality (12) follows, and Theorem 2.3 is proved.

Corollary 2.3. Under conditions of Theorem 2.3, if $s=1$, then

$$
\begin{gathered}
\left|\int_{a}^{b} f(x) g(x) d x-f\left(\frac{a+b}{2}\right) \int_{a}^{b} g(x) d x\right| \leq \\
\leq \frac{(b-a)^{2}}{4^{1+1 / q}}|| g \|_{\infty}\left(\frac{q-1}{2 q-1}\right)^{1-1 / q} \times \\
\times\left\{\left[3\left|f^{\prime}(a)\right|^{q}+\left|f^{\prime}(b)\right|^{q}\right]^{1 / q}+\left[\left|f^{\prime}(a)\right|^{q}+3\left|f^{\prime}(b)\right|^{q}\right]^{1 / q}\right\} .
\end{gathered}
$$

Theorem 2.4. Let $f: I \subseteq \mathbf{R} \rightarrow \mathbf{R}$ be differentiable on int $I$ and $g:[a, b] \rightarrow[0, \infty)$ be continuous and symmetric to $\frac{a+b}{2}$ for $a, b \in I$ with $a<b$ such that $f^{\prime} \in L^{1}[a, b]$. If $\left|f^{\prime}\right|^{q}$ is s-convex on $[a, b]$ for $q>1$ and some fixed $s \in(0,1]$, then

$$
\begin{aligned}
& \left|\int_{a}^{b} f(x) g(x) d x-f\left(\frac{a+b}{2}\right) \int_{a}^{b} g(x) d x\right| \leq \frac{(b-a)^{2}}{4(s+1)^{\frac{1}{q}}}\left(\frac{q-1}{2 q-1}\right)^{1-\frac{1}{q}}\|g\|_{\infty} \times \\
& \quad \times\left\{\left[\left|f^{\prime}(a)\right|^{q}+\left|f^{\prime}\left(\frac{a+b}{2}\right)\right|^{q}\right]^{1 / q}+\left[\left|f^{\prime}\left(\frac{a+b}{2}\right)\right|^{q}+\left|f^{\prime}(b)\right|^{q}\right]^{1 / q}\right\} .
\end{aligned}
$$

Proof. Notice that $\left|f^{\prime}\right|^{q}$ is s-convex on $[a, b]$, by (4) in Lemma 2.1 with the second equality in (1) and (2), and using the Hölder inequality, we have

$$
\begin{gathered}
\left|\int_{a}^{b} f(x) g(x) d x-f\left(\frac{a+b}{2}\right) \int_{a}^{b} g(x) d x\right| \leq \\
\leq \frac{(b-a)^{2}}{4}\|g\|_{\infty} \int_{0}^{1}(1-t)\left[\left|f^{\prime}(L(t))\right|+\left|f^{\prime}(U(t))\right|\right] d t=\frac{(b-a)^{2}}{4}\|g\|_{\infty} \times \\
\times \int_{0}^{1}(1-t)\left[\left|f^{\prime}\left(t a+(1-t) \frac{a+b}{2}\right)\right|+\left|f^{\prime}\left(t b+(1-t) \frac{a+b}{2}\right)\right|\right] d t \leq
\end{gathered}
$$




$$
\begin{gathered}
\leq \frac{(b-a)^{2}}{4}\|g\|_{\infty}\left[\int_{0}^{1}(1-t)^{q / q-1} d t\right]^{1-1 / q} \times \\
\times\left\{\left[\int_{0}^{1}\left(t^{s}\left|f^{\prime}(a)\right|^{q}+(1-t)^{s}\left|f^{\prime}\left(\frac{a+b}{2}\right)\right|^{q}\right) d t\right]^{1 / q}+\right. \\
\left.+\left[\int_{0}^{1}\left((1-t)^{s}\left|f^{\prime}\left(\frac{a+b}{2}\right)\right|^{q}+t^{s}\left|f^{\prime}(b)\right|^{q}\right) d t\right]^{1 / q}\right\}= \\
=\frac{(b-a)^{2}}{4}\|g\|_{\infty}\left(\frac{q-1}{2 q-1}\right)^{1-1 / q}\left(\frac{1}{s+1}\right)^{1 / q}\left\{\left[\left|f^{\prime}(a)\right|^{q}+\right.\right. \\
\left.\left.+\left|f^{\prime}\left(\frac{a+b}{2}\right)\right|^{q}\right]^{1 / q}+\left[\left|f^{\prime}\left(\frac{a+b}{2}\right)\right|^{q}+\left|f^{\prime}(b)\right|^{q}\right]^{1 / q}\right\} .
\end{gathered}
$$

Inequality (13) follows, and Theorem 2.4 is proved.

Corollary 2.4. Under conditions of Theorem 2.4, if $s=1$, then

$$
\begin{gathered}
\left|\int_{a}^{b} f(x) g(x) d x-f\left(\frac{a+b}{2}\right) \int_{a}^{b} g(x) d x\right| \leq \\
\leq \frac{(b-a)^{2}}{2^{2+1 / q}}|| g \|_{\infty}\left(\frac{q-1}{2 q-1}\right)^{1-1 / q} \times \\
\times\left\{\left[\left|f^{\prime}(a)\right|^{q}+\left|f^{\prime}\left(\frac{a+b}{2}\right)\right|^{q}\right]^{1 / q}+\left[\left|f^{\prime}\left(\frac{a+b}{2}\right)\right|^{q}+\left|f^{\prime}(b)\right|^{q}\right]^{1 / q}\right\} .
\end{gathered}
$$

Theorem 2.5. Let $f: I \subseteq \mathbf{R} \rightarrow \mathbf{R}$ be differentiable on int $I$ and $g:[a, b] \rightarrow[0, \infty)$ be continuous and symmetric to $\frac{a+b}{2}$ for $a, b \in I$ with $a<b$. If $\left|f^{\prime}\right|^{q}$ for $q \geq 1$ is convex on $[a, b]$, then

$$
\left|\int_{a}^{b} f(x) g(x) d x-f\left(\frac{a+b}{2}\right) \int_{a}^{b} g(x) d x\right| \leq
$$




$$
\leq(b-a)\left[\frac{\left|f^{\prime}(a)\right|^{q}+\left|f^{\prime}(b)\right|^{q}}{2}\right]^{1 / q} \int_{0}^{1}\left[\int_{a}^{L(t)} g(x) d x\right] d t .
$$

Proof. Notice that $\left|f^{\prime}\right|^{q}$ is convex on $[a, b]$, by (4) in Lemma 2.1 and using the Hölder inequality, we have

$$
\begin{gathered}
\left|\int_{a}^{b} f(x) g(x) d x-f\left(\frac{a+b}{2}\right) \int_{a}^{b} g(x) d x\right| \leq \frac{b-a}{2} \times \\
\times\left\{\int_{0}^{1}\left[\int_{a}^{L(t)} g(x) d x\right]\left|f^{\prime}(L(t))\right| d t+\int_{0}^{1}\left[\int_{a}^{L(t)} g(x) d x\right]\left|f^{\prime}(U(t))\right| d t\right\} \leq \\
\leq \frac{b-a}{2}\left\{\int_{0}^{1}\left[\int_{a}^{L(t)} g(x) d x\right] d t\right\}^{1-\frac{1}{q}}\left\{\left(\int_{0}^{1}\left[\int_{a}^{L(t)} g(x) d x\right]\left|f^{\prime}(L(t))\right|^{q} d t\right)^{\frac{1}{q}}+\right. \\
\left.+\left(\int_{0}^{1}\left[\int_{a}^{L(t)} g(x) d x\right]\left|f^{\prime}(U(t))\right|^{q} d t\right)^{\frac{1}{q}}\right\} .
\end{gathered}
$$

From the power-mean inequality $\left(a^{r}+b^{r}\right) \leq 2^{1-r}(a+b)^{r}$ for $a, b>0$ and $r \leq 1$ and convexity of $\left|f^{\prime}\right|^{q}$ on $[a, b]$, with the second equality in (1) and (2), we obtain

$$
\begin{aligned}
& \left(\int_{0}^{1}\left[\int_{a}^{L(t)} g(x) d x\right]\left|f^{\prime}(L(t))\right|^{q} d t\right)^{\frac{1}{q}}+\left(\int_{0}^{1}\left[\int_{a}^{L(t)} g(x) d x\right]\left|f^{\prime}(U(t))\right|^{q} d t\right)^{\frac{1}{q}} \leq \\
& \leq 2^{1-1 / q}\left\{\int_{0}^{1}\left[\int_{a}^{L(t)} g(x) d x\right]\left[\left|f^{\prime}(L(t))\right|^{q}+\left|f^{\prime}(U(t))\right|^{q}\right] d t\right\}^{1 / q} \leq 2^{1-1 / q} \times \\
& \times\left\{\int_{0}^{1}\left[\int_{a}^{L(t)} g(x) d x\right]\left[t\left|f^{\prime}(a)\right|^{q}+2(1-t)\left|f^{\prime}\left(\frac{a+b}{2}\right)\right|^{q}+t\left|f^{\prime}(b)\right|^{q}\right] d t\right\}^{1 / q} \leq
\end{aligned}
$$




$$
\begin{aligned}
& \leq 2^{1-1 / q}\left\{\int _ { 0 } ^ { 1 } [ \int _ { a } ^ { L ( t ) } g ( x ) d x ] \left[t\left|f^{\prime}(a)\right|^{q}+(1-t)\left(\left|f^{\prime}(a)\right|^{q}+\left|f^{\prime}(b)\right|^{q}\right)+t \times\right.\right. \\
& \left.\left.\times\left|f^{\prime}(b)\right|^{q}\right] d t\right\}^{\frac{1}{q}}=2^{1-\frac{1}{q}}\left[\left|f^{\prime}(a)\right|^{q}+\left|f^{\prime}(b)\right|^{q}\right]^{\frac{1}{q}}\left\{\int_{0}^{1} \int_{a}^{L(t)} g(x) d x d t\right\}^{\frac{1}{q}} \cdot
\end{aligned}
$$

Inequality (14) follows from (15) and (16), and Theorem 2.5 is proved.

Corollary 2.5.1. Under conditions of Theorem 2.5, if $q=1$, then

$$
\begin{aligned}
& \left|\int_{a}^{b} f(x) g(x) d x-f\left(\frac{a+b}{2}\right) \int_{a}^{b} g(x) d x\right| \leq \\
& \leq \frac{b-a}{2}\left[\left|f^{\prime}(a)\right|+\left|f^{\prime}(b)\right|\right] \int_{0}^{1}\left[\int_{a}^{L(t)} g(x) d x\right] d t .
\end{aligned}
$$

Theorem 2.6. Let $f: I \subseteq \mathbf{R} \rightarrow \mathbf{R}$ be differentiable on int $I$ and $g:[a, b] \rightarrow[0, \infty)$ be continuous and symmetric to $\frac{a+b}{2}$ for $a, b \in I$ with $a<b$. If $\left|f^{\prime}\right|^{q}$ for $q>1$ is convex on $[a, b]$, then

$$
\begin{gathered}
\left|\int_{a}^{b} f(x) g(x) d x-f\left(\frac{a+b}{2}\right) \int_{a}^{b} g(x) d x\right| \leq \\
\leq \frac{b-a}{2}\left\{\left[\frac{3\left|f^{\prime}(a)\right|^{q}+\left|f^{\prime}(b)\right|^{q}}{4}\right]^{1 / q}+\left[\frac{\left|f^{\prime}(a)\right|^{q}+3\left|f^{\prime}(b)\right|^{q}}{4}\right]^{1 / q}\right\} \times \\
\times\left\{\int_{0}^{1}\left[\int_{a}^{L(t)} g(x) d x\right]^{q / q-1} d t\right\}^{1-1 / q} .
\end{gathered}
$$

Proof. Notice that $\left|f^{\prime}\right|^{q}$ is convex on $[a, b]$, by (4) in Lemma 2.1 with the second equality in (1) and (2), and using the Hölder inequality, we have

$$
\left|\int_{a}^{b} f(x) g(x) d x-f\left(\frac{a+b}{2}\right) \int_{a}^{b} g(x) d x\right| \leq \frac{b-a}{2}\left\{\int_{0}^{1}\left[\int_{a}^{L(t)} g(x) d x\right] \times\right.
$$




$$
\begin{aligned}
& \left.\times\left[\left|f^{\prime}(L(t))\right|+\left|f^{\prime}(U(t))\right|\right] d t\right\} \leq \frac{b-a}{2}\left\{\int_{0}^{1}\left[\int_{a}^{L(t)} g(x) d x\right]^{\frac{q}{q-1}} d t\right\}^{1-\frac{1}{q}} \times \\
& \times\left\{\left[\int_{0}^{1}\left|f^{\prime}(L(t))\right|^{q} d t\right]^{\frac{1}{q}}+\left[\int_{0}^{1}\left|f^{\prime}(U(t))\right|^{q} d t\right]^{\frac{1}{q}}\right\} \leq \frac{b-a}{2} \times \\
& \times\left\{\int_{0}^{1}\left[\int_{a}^{L(t)} g(x) d x\right]^{\frac{q}{q-1}} d t\right\}^{1-\frac{1}{q}}\left\{\left(\int _ { 0 } ^ { 1 } \left[t\left|f^{\prime}(a)\right|^{q}+(1-t) \times\right.\right.\right. \\
& \left.\left.\left.\times\left|f^{\prime}\left(\frac{a+b}{2}\right)\right|^{q}\right] d t\right)^{\frac{1}{q}}+\left(\int_{0}^{1}\left[(1-t)\left|f^{\prime}\left(\frac{a+b}{2}\right)\right|^{q}+t\left|f^{\prime}(a)\right|^{q}\right] d t\right)^{\frac{1}{q}}\right\} \leq \\
& \leq \frac{b-a}{2}\left\{\int_{0}^{1}\left[\int_{a}^{L(t)} g(x) d x\right]^{\frac{q}{q-1}} d t\right\}^{1-\frac{1}{q}}\left\{\left(\int _ { 0 } ^ { 1 } \left[\frac{1+t}{2}\left|f^{\prime}(a)\right|^{q}+\right.\right.\right. \\
& \left.\left.\left.+\frac{1-t}{2}\left|f^{\prime}(b)\right|^{q}\right] d t\right)^{\frac{1}{q}}+\left(\int_{0}^{1}\left[\frac{1-t}{2}\left|f^{\prime}(a)\right|^{q}+\frac{1+t}{2}\left|f^{\prime}(b)\right|^{q}\right] d t\right)^{\frac{1}{q}}\right\}= \\
& =\frac{b-a}{2}\left\{\left[\frac{3\left|f^{\prime}(a)\right|^{q}+\left|f^{\prime}(b)\right|^{q}}{4}\right]^{\frac{1}{q}}+\left[\frac{\left|f^{\prime}(a)\right|^{q}+3\left|f^{\prime}(b)\right|^{q}}{4}\right]^{\frac{1}{q}}\right\} \times \\
& \times\left\{\int_{0}^{1}\left[\int_{a}^{L(t)} g(x) d x\right]^{\frac{q}{q-1}} d t\right\}^{1-\frac{1}{q}}
\end{aligned}
$$

Inequality (17) follows, and Theorem 2.6 is proved.

3. Applications to special means. Now we apply some of the above inequalities of Hermite-Hadamard type involving the product of an s-convex function and a symmetric function to construct inequalities for special means.

For positive numbers $a>0$ and $b>0$, define

(i) the arithmetic mean:

$$
A(a, b)=\frac{a+b}{2}
$$


and

(ii) the generalized logarithmic mean:

$$
L_{r}(a, b)=\left[\frac{b^{r+1}-a^{r+1}}{(r+1)(b-a)}\right]^{1 / r}, r \neq-1,0 .
$$

Let

$$
f(x)=\frac{x^{s+1}}{s+1}
$$

for $x>0, s>0$, and $q \geq 1$. If $0<s q \leq 1$ and $0<s \leq 1$, we have

$$
\left|f^{\prime}(t x+(1-t) y)\right|^{q} \leq t^{s q} x^{s q}+(1-t)^{s q} y^{s q} \leq t^{s}\left|f^{\prime}(x)\right|^{q}+(1-t)^{s}\left|f^{\prime}(y)\right|^{q}
$$

for $x, y>0$ and $t \in[0,1]$. At this time, it is easy to verify that the function $\left|f^{\prime}(x)\right|^{q}=x^{s q} \in K_{s}^{2}$ for $x \in[a, b]$.

For $b>a>0$, define

$$
g(x)=\left(x-\frac{a+b}{2}\right)^{2}
$$

for $x \in[a, b]$. Applying Theorems 2.1-2.4 to the concrete functions (18) and (19) straightforwardly yields the following inequalities involving special means $A$ and $L_{r}$.

Theorem 3.1. For $b>a>0, q \geq 1$, and $0<s \leq 1$ such that $0<s q \leq 1$, we have

$$
\begin{aligned}
& \mid(b-a)^{2} A^{s+1}(a, b)-12\left[L_{s+3}^{s+3}(a, b)-2 A(a, b) L_{s+2}^{s+2}(a, b)+\right. \\
& \left.+A^{2}(a, b) L_{s+1}^{s+1}(a, b)\right] \mid \leq \frac{3 \times 2^{(1-s) / q}(b-a)^{3}(s+1)^{1-1 / q}}{8(s+2)^{1 / q}} \times
\end{aligned}
$$

$\times\left\{\left[\left(2^{s+2}-s-3\right) a^{s q}+(s+1) b^{s q}\right]^{1 / q}+\left[(s+1) a^{s q}+\left(2^{s+2}-s-3\right) b^{s q}\right]^{1 / q}\right\}$ and

$$
\begin{aligned}
& \mid(b-a)^{2} A^{s+1}(a, b)-12\left[L_{s+3}^{s+3}(a, b)-2 A(a, b) L_{s+2}^{s+2}(a, b)+\right. \\
& \left.+A^{2}(a, b) L_{s+1}^{s+1}(a, b)\right] \mid \leq \frac{3 \times 2^{1 / q}(b-a)^{3}(s+1)^{1-1 / q}}{8(s+2)^{1 / q}} \times
\end{aligned}
$$




$$
\times\left\{\left[a^{s q}+(s+1) A^{s q}(a, b)\right]^{1 / q}+\left[(s+1) A^{s q}(a, b)+b^{s q}\right]^{1 / q}\right\} .
$$

Corollary 3.1.1. For $b>a>0$ and $0<s \leq 1$, we have

$$
\begin{aligned}
& \mid(b-a)^{2} A^{s+1}(a, b)-12\left[L_{s+3}^{s+3}(a, b)-2 A(a, b) L_{s+2}^{s+2}(a, b)+\right. \\
& \left.\quad+A^{2}(a, b) L_{s+1}^{s+1}(a, b)\right] \mid \leq \frac{3(b-a)^{3}}{2^{s+1}(s+2)}\left(2^{s+1}-1\right)\left(a^{s}+b^{s}\right)
\end{aligned}
$$

and

$$
\begin{gathered}
\mid(b-a)^{2} A^{s+1}(a, b)-12\left[L_{s+3}^{s+3}(a, b)-2 A(a, b) L_{s+2}^{s+2}(a, b)+\right. \\
\left.+A^{2}(a, b) L_{s+1}^{s+1}(a, b)\right] \mid \leq \frac{3(b-a)^{3}}{2(s+2)}\left[A\left(a^{s}, b^{s}\right)+(s+1) A^{s}(a, b)\right] .
\end{gathered}
$$

Theorem 3.2. For $b>a>0, q>1$, and $0<s<1$ such that $0<s q \leq 1$, we have

$$
\begin{aligned}
& \mid(b-a)^{2} A^{s+1}(a, b)-12\left[L_{s+3}^{s+3}(a, b)-2 A(a, b) L_{s+2}^{s+2}(a, b)+\right. \\
& \left.+A^{2}(a, b) L_{s+1}^{s+1}(a, b)\right] \mid \leq \frac{3(b-a)^{3}(s+1)^{1-1 / q}}{2^{2+s / q}}\left(\frac{q-1}{2 q-1}\right)^{1-1 / q} \times \\
& \times\left\{\left[\left(2^{s+1}-1\right) a^{s q}+b^{s q}\right]^{1 / q}+\left[a^{s q}+\left(2^{s+1}-1\right) b^{s q}\right]^{1 / q}\right\}
\end{aligned}
$$

and

$$
\begin{gathered}
\mid(b-a)^{2} A^{s+1}(a, b)-12\left[L_{s+3}^{s+3}(a, b)-2 A(a, b) L_{s+2}^{s+2}(a, b)+\right. \\
\left.+A^{2}(a, b) L_{s+1}^{s+1}(a, b)\right] \mid \leq \frac{3(b-a)^{3}(s+1)^{1-1 / q}}{4}\left(\frac{q-1}{2 q-1}\right)^{1-1 / q} \times \\
\times\left\{\left[a^{s q}+A^{s q}(a, b)\right]^{1 / q}+\left[A^{s q}(a, b)+b^{s q}\right]^{1 / q}\right\} .
\end{gathered}
$$

Acknowledgment. The author would like to thank the referees for their helpful comments and suggestions. 


\section{References}

[1] Hudzik H., Maligranda L. Some remarks on s-convex functions. Aequationes Mathmaticae, 1994, vol. 17, no. 2, pp. 100-111.

[2] Hua J., Xi B. Y., Qi F. Inequalities of Hermite-Hadamard type involving an s-convex function with applications. Appl. Math. Comput., 2014, vol. 246, pp. $752-760$.

[3] Hwang D. Y. Some inequalities for differentiable convex mapping with application to weighted midpoint formula and higher moments of random variables. Appl. Math. Comput., 2014, vol. 232, pp. 68-75.

[4] Pearce C. E. M., Pečarić J. Inequalities for differentiable mappings with applications to special means and quadrature formula. Appl. Math. Lett., 2000, vol. 13, pp. 51-55.

[5] Yang G. S., Hwang D. Y., Kseng K. I. Some inequalities for differentiable convex and concave mappings. Computer and Mathematics with Applications, 2004, vol. 47, pp. 207-216.

Received March 27, 2016.

In revised form, June 22, 2016.

Accepted June 15, 2016.

University of Science and Technology Liaoning

Institute of Applied Mathematics, School of Science

Anshan 114051, Liaoning, China;

Room 2004, 4 Lane 123

Guiping Road, Shanghai 200233, China

E-mail: lewzheng@163.net 